\title{
LA ESTRATEGIA DE LAS PEQUEÑAS EMPRESAS INDUSTRIALES EN LA REGIÓN LIMA
}

\author{
THE STRATEGY OF SMALL INDUSTRIAL ENTERPRISES IN THE LIMA REGION \\ Luis Alberto Baldeos Ardian', Flor de Maria Lioo Jordan', \\ Flor de Maria Garivay Torres ${ }^{1}$
}

\begin{abstract}
RESUMEN
Objetivo: Determinar cómo la falta de estrategias genera una baja rentabilidad de las Pequeñas Empresas Industriales en la Región Lima. Métodos: La población estuvo compuesta por 60 Pequeñas Empresas Industriales y se tomó una muestra aleatoria de 28 pequeñas empresas Industriales de la Región Lima. Se utilizaron instrumentos de medición los que han sido validados por expertos. La investigación es de nivel Descriptivo - Explicativo. Resultados: El $21.43 \%$ de los Gerentes conocen, el $71.42 \%$ no conocen y el $7.15 \%$ poco conocen, es decir la falta de estrategias influye en el bajo rendimiento de las Pequeñas Empresas Industriales. El $17.9 \%$ de Gerentes conocen, el $67.9 \%$ no conocen y el 14.2 poco conocen, que la falta de estrategias genera el bajo rendimiento del personal. El $35.7 \%$ de los Gerentes conocen, el $42.9 \%$ no conocen y el $21.4 \%$ poco conocen, que la falta de estrategias genera productos y servicios no competitivos. El 21.4 de los Gerentes conocen, el 60.7 no conocen y el 17,9 poco conocen, que la falta de estrategias de las pequeñas empresas industriales de la Región Lima influye en el PBI Nacional. Conclusiones: Se ha comprobado que las Pequeñas Empresas Industriales de la Región Lima, por la Falta de Estrategias tienen un bajo rendimiento y se elevan sus costos; así como también baja el rendimiento del personal y hay un escaso interés de los pequeños empresarios industriales para buscar la competitividad de sus productos y servicios.
\end{abstract}

Palabras clave: Estrategias, Competitividad, Rentabilidad.

\begin{abstract}
Objective: To determine how the lack of Strategies generates a low profitability of the Small Industrial Companies in the Lima Region. Methods: The population was composed of 60 Small Industrial Enterprises and a random sample of 28 small Industrial companies of the Lima Region was taken. Measured instruments were used and validated by experts. The research is descriptive - Explanatory level. Results: $21.43 \%$ of managers know, $71.42 \%$ do not know and $7.15 \%$ do not know much, that is to say, the lack of strategies influences the low performance of small industrial companies. The $17.9 \%$ of managers know, $67.9 \%$ do not know and 14.2 little know, that the lack of strategies generates the low performance of the staff. $35.7 \%$ of the Managers know, $42.9 \%$ do not know and $21.4 \%$ do not know much, that the lack of strategies generates non-competitive products and services. 21.4 of the Managers know, 60.7 do not know and 17.9 little know, that the lack of strategies of small industrial companies in the Lima Region influences the National GDP. Conclusions: It has been verified that the Small Industrial Companies of the Lima Region, due to the Lack of Strategies, have a low performance and raise their costs; as well as low staff performance and there is little interest of small industrial entrepreneurs to seek the competitiveness of their products and services.
\end{abstract}

keywords: Strategies, Competitiveness, Profitabilit.

'Facultad de Ciencias Empresariales, Universidad Nacional José Faustino Sánchez Carrión. Email: Ibaldeos@yahoo.es 


\section{INTRODUCCIÓN}

Las pequeñas empresas industriales en el Perú y en particular de la Región Lima están pasando una crisis de estancamiento empresarial, donde sus posibilidades de crecimiento son casi nula. Dando lugar a que estas pueden tender a que fracasen y desaparezcan de la economía nacional. Uno de los grandes problemas es la informalidad de estas empresas por los altos impuesto y otros gravámenes así como por el alto costo laboral y esto es muy preocupante por la alta incidencia en la falta de competitividad empresarial de este sector. También es preocupante la falta de interés del Estado para solucionar este gran problema que está generando la falta de empleo y la escasa contribución al PBI Nacional.

Cuervo García (1999): Afirma que la Dirección Estratégica necesita algo más que relaciones estadísticas entre retornos anormales, medidos por rentabilidades de mercado, y medidas estratégicas como consecuencia de investigaciones sobre la ventaja competitiva.

Mintzberg y Lampel (1999): La Estrategia ha tenido durante mucho tiempo sus distinciones históricas; afortunadamente, está experimentando un eclecticismo recién descubierto". Sugerido que los procesos de dirección estratégica son, o deberían ser, procesos analíticos secuencialmente racionales. Otros defienden que los decisores tienen capacidad limitada para procesar la información y las organizaciones son entidades políticas con intereses individuales o de grupo que determinan su actividad.

Pettigrew et al. (2002): Considerando sus apreciaciones que la historia de la Dirección Estratégica es un relato de adopciones promiscuas de otras disciplinas y sub campos de la dirección de empresas.

La falta de Estrategias en las Pequeñas Empresas Industriales en la Región Lima, genera baja Rentabilidad, bajo Rendimiento del Personal, fabricación de productos y servicios no competitivos e influye en la baja contribución al PBI nacional.

\section{MATERIALES Y MÉTODOS}

La investigación es del tipo aplicativa, con un nivel Descriptivo - Explicativo, se ha utilizado como instrumentos una encuesta y un cuestionario. Los encuestados han sido los Gerentes de las Pequeñas Empresas Industriales en la Region Lima, su desarrollo les permitió responder a las interrogantes y objetivos de la investigación. Se utilizó los conocimientos de las variables como la estrategia y el rendimiento empresarial; así como también la estadística descriptiva lo que nos facilitó describir sus características de las variables a través de métodos gráficos los que han sido validados por juicio de expertos.

Para contrastar la hipótesis general se utilizó la regresión logística y para las hipótesis especificas se ha utilizado la prueba no paramétrica chi-cuadrado.

El análisis estadístico se realizó con el programa SPSS versión 20. La población estuvo compuesta por 60 Pequeñas Empresas Industriales de la Region Lima y se tomó una muestra aleatoria de 28 Pequeñas Empresas Industriales. Se tomó un muestreo aleatorio simple con un nivel de confianza del 95\% esta muestra constituida por 28 Pequeñas Empresas Industriales de la Región Lima.

\section{RESULTADOS}

En lo que respecta a la falta de estrategias y el bajo rendimiento de las pequeñas empresas industriales de la región, el $21 \%$ de los gerentes es decir solo 06 gerentes conocen sobre la relación que tienen la falta de estrategias en el bajo rendimiento de las pequeñas empresas industriales de la Región Lima.

Así mismo, el $71 \%$, es decir 20 gerentes de 28 no conocen sobre la importancia que tienen las estrategias para que las pequeñas empresas industriales tengan un buen rendimiento y el $7.1 \%$ poco conoce o sea que las pequeñas empresas industriales tienen un bajo rendimiento por sus altos costos.

Tabla 1. Cruzada Falta De Estrategias* Bajo Rendimiento De Pequeñas Empresas Industriales

\begin{tabular}{|l|c|c|c|c|}
\hline \multirow{2}{*}{$\begin{array}{l}\text { FALTA DE } \\
\text { ESTRATEGIAS }\end{array}$} & $\begin{array}{l}\text { BAJO RENDIMIENTO DE PEQUENAS EMPRESAS } \\
\text { INDUSTRIALES }\end{array}$ & \multirow{2}{*}{ TOTAL } \\
\cline { 2 - 5 } & CONOCE & NO CONOCE & POCO CONOCE & \\
\hline CONOCE & $21,4 \%$ & $0,0 \%$ & $0,0 \%$ & $21,4 \%$ \\
\hline NO CONOCE & $0,0 \%$ & $71,4 \%$ & $0,0 \%$ & $71,4 \%$ \\
\hline POCO CONOCE & $0,0 \%$ & $0,0 \%$ & $7,1 \%$ & $7,1 \%$ \\
\hline \multicolumn{1}{|l|}{ TOTAL } & $21,4 \%$ & $71.4 \%$ & $7,1 \%$ & $100,0 \%$ \\
\hline
\end{tabular}

Al respecto solo $17.86 \%$ es decir 5 gerentes de 28 conocen la importancia que tienen las estrategias para mejorar el rendimiento del personal de las pequeñas empresas. El $67.8 \%$, es decir 19 gerentes de los 28 consultados argumentan que no conocen sobre la importancia que tienen las estrategias para mejorar el rendimiento del personal y el $14 \%$ de los gerentes respondieron que poco conocen sobre el tema en particular, lo cual explica que hay un bajo rendimiento no solo del personal sino también de los gerentes de las pequeñas empresas industriales de la Región Lima.

Tabla 2. Cruzada Falta De Estrategias* Bajo Rendimiento Del Personal Pequeñas Empresas Industriales

\begin{tabular}{|l|c|c|c|c|}
\hline \multirow{2}{*}{$\begin{array}{l}\text { FALTA DE } \\
\text { ESTRATEGIAS }\end{array}$} & \multicolumn{3}{|l|}{$\begin{array}{l}\text { BAJO RENDIMIENTO DE PEQUENAS EMPRESAS } \\
\text { INDUSTRIALES }\end{array}$} & \multirow{2}{*}{ TOTAL } \\
& \multicolumn{3}{l|}{} & \\
\cline { 2 - 5 } & CONOCE & NO CONOCE & POCO CONOCE & \\
\hline CONOCE & $17,9 \%$ & $3,6 \%$ & $0,0 \%$ & $21,4 \%$ \\
\hline NO CONOCE & $0,0 \%$ & $64,3 \%$ & $7,1 \%$ & $71,4 \%$ \\
\hline POCO CONOCE & $0,0 \%$ & $0,0 \%$ & $7,1 \%$ & $7,1 \%$ \\
\hline \multicolumn{1}{|c|}{ TOTAL } & $17,9 \%$ & $67,9 \%$ & $14,3 \%$ & $100,0 \%$ \\
\hline
\end{tabular}

El $36 \%$ aproximadamente o 10 gerentes de estas empresas han respondido que si conocen sobre esta relación entre estrategias y la fabricación de productos no competitivos. El $43 \%$ aproximadamente respondieron que no conocen esta relación entre las estrategias y la falta competitividad de sus productos y servicios. Por último, el $21 \%$ de los gerentes conocen poco sobre esta relación.

Tabla 3. Cruzada Falta De Estrategias* Fabricación De Productos Y Servicios No competitivos De Las Pequeñas Empresas Industriales 


\begin{tabular}{|l|c|c|c|c|}
\hline \multirow{2}{*}{$\begin{array}{l}\text { FALTA DE } \\
\text { ESTRATEGIAS }\end{array}$} & \multicolumn{3}{|l|}{$\begin{array}{l}\text { BAJO RENDIMIENTO DE } \\
\text { NDDUSTRIALES }\end{array}$} & \multirow{2}{*}{ TOTAL } \\
\cline { 2 - 4 } & CONOCE & NO CONOCE & POCO CONOCE & \\
\hline CONOCE & $21,4 \%$ & $0,0 \%$ & $0,0 \%$ & $21,4 \%$ \\
\hline NO CONOCE & $14,3 \%$ & $42,9 \%$ & $14,3 \%$ & $71,4 \%$ \\
\hline POCO CONOCE & $0,0 \%$ & $0,0 \%$ & $7,1 \%$ & $7,1 \%$ \\
\hline TOOTAL & $35,7 \%$ & $42,9 \%$ & $21,4 \%$ & $100,0 \%$ \\
\hline
\end{tabular}

El $21 \%$ de los gerentes conocen sobre esta relación entre estrategias y el PBI Nacional, el 71\% no conocen y el $7.1 \%$ poco conocen, que la falta de estrategias de las Pequeñas Empresas Industriales de la Región Lima influyen en el PBI Nacional.

Tabla 4. Cruzada Falta De Estrategias* PBI Nacional

\begin{tabular}{|l|c|c|c|c|}
\hline \multirow{3}{*}{$\begin{array}{l}\text { FALTA DE } \\
\text { ESTRATEGIAS }\end{array}$} & \multicolumn{3}{|l|}{$\begin{array}{l}\text { BAJO RENDIMIENTO DE PEQUENAS EMPRESAS } \\
\text { INDUSTRIALES }\end{array}$} & \multirow{2}{*}{ TOTAL } \\
\cline { 2 - 4 } & CONOCE & NO CONOCE & POCO CONOCE & \\
\hline CONOCE & $21,4 \%$ & $0,0 \%$ & $0,0 \%$ & $21,4 \%$ \\
\hline NO CONOCE & $0,0 \%$ & $60,7 \%$ & $10,7 \%$ & $71,4 \%$ \\
\hline POCO CONOCE & $0,0 \%$ & $0,0 \%$ & $7,1 \%$ & $7,1 \%$ \\
\hline TOTAL & $21,4 \%$ & $60,7 \%$ & $17,9 \%$ & $100,0 \%$ \\
\hline
\end{tabular}

Tabla 5. Pruebas De Chi Cuadrado

\begin{tabular}{|l|c|c|c|}
\hline & Valor & gl & Significación asintótica (bilateral) \\
\hline Chi-cuadrado de Pearson & 37,520 & 4 & 0,000 \\
\hline Razón de verosimilitud & 35,770 & 4 & 0,000 \\
\hline N de casos válidos & 28 & & \\
\hline
\end{tabular}

Como se observa la significancia asintótica 0,00 es menor que el nivel de significación 0.05 , se acepta la hipótesis.

\section{DISCUSIÓN}

En lo que respecta a la falta de estrategias y el bajo rendimiento de las pequeñas empresas industriales de la Región Lima, el $21 \%$ de los gerentes es decir 06 gerentes si conocen sobre la relación que tienen la falta de estrategias en el bajo rendimiento de las pequeñas empresas.

El $71 \%$, es decir 20 gerentes de 28 no conocen sobre la importancia que tienen las estrategias para que las pequeñas empresas industriales de la Región Lima tengan un buen rendimiento.

El $17.86 \%$ es decir 5 gerentes de 28 conocen la importancia que tienen las estrategias para mejorar el rendimiento del personal de las pequeñas empresas industriales de la Región Lima. Estos gerentes se han preocupado en capacitarse sobre el tema y debido a esta situación es que han mejorado el rendimiento de su personal.

Un alto porcentaje de los gerentes, es decir $67.8 \%$, es decir 19 gerentes de los 28 consultados argumentan que no conocen sobre la importancia que tienen las estrategias para mejorar el rendimiento del personal de las pequeñas empresas industriales de la Región Lima.

Los gerentes, el 68\% aproximadamente argumenta que no tienen tiempo para capacitarse sobre las estrategias empresariales, pero si están preocupados sobre el bajo rendimiento del personal.

El $14 \%$ de los gerentes respondieron que poco conocen sobre el tema en particular, lo cual explica que hay un bajo rendimiento no solo del personal sino también de los gerentes de las pequeñas empresas industriales de la Región Lima.

El $36 \%$ aproximadamente o 10 gerentes de estas empresas han respondido que si conocen sobre esta relación entre estrategias y la fabricación de productos no competitivos.

El $43 \%$ aproximadamente respondieron que no conocen esta relación entre las estrategias y la falta competitividad de sus productos y servicios. Esto se caracteriza, debido a que estos empresarios no han buscado que vender al exterior y no tiene conocimiento sobre las estrategias y la competitividad de sus productos.

El $21 \%$ de los gerentes conocen poco sobre esta relación. Se ha concluido que hay un escaso interés de estos empresarios para buscar la competitividad de sus productos.

El $21.4 \%$ de los gerentes conocen, el $60.7 \%$ no conocen y el $17,9 \%$ poco conocen, que la falta de estrategias de las Pequeñas Empresas Industriales de la Región Lima influye en el PBI Nacional.

\section{REFERENCIAS BIBLIOGRÁFICA}

Arias, Fernando L. Galicia; Heredia, Víctor Espinosa. (2004). "Administración de Recursos Humanos para el Alto Desempeño". 3a. Edición. México: Editorial Trillas.

Bateman, Thomas S. Y Scott A. Snell. (2001). Administración: una ventaja competitiva. Ed. McGraw-Hill. México.

Berry, Leonard L., David R. Bennet. Y Carter W. Brown (1989). Calidad de Servicio. Serie liderazgo en Banca. Madrid.

Certo, Samuel C. (2001): Administración Moderna. 8va edición. Editorial Pearson Educación Colombiana. Ltda.

Crosby Philip (2000). Hablemos de Calidad. Editorial McGraw-Hill. 5ta Edición. Madrid.

Chiavenato, Idalberto. $(1,999)$ Administración de Recursos Humanos. Editorial Mc Graw-Hill Interamericana S.A. 5ta Edición Colombia.

Chiavenato, Idalberto (2006). Introducción a la Teoría General de la Administración. Ed. McGraw-Hill. México.

Daft, Richard. (2,004). La Administración. Editorial Thomson Editores, S.A.de C.V. 6ta Edición México.

Doch, Iván. (2,000). Estrategia Empresarial en una Economía de Competitividad Editorial Gráfica Gago SRL. Perú.

Don Tapscott, Art Caston $(1,995)$ Cambio de Paradigma Empresariales Editorial Lerner Ltda. Colombia.

Drucker, Peter F. $(1,999)$ Los desafíos de la Administración en el siglo XXI. Editorial Sudamericana. Argentina.

Davis, Keith; Newstrom, John W. (2000). "Comportamiento Humano en el Trabajo". 10a Edición. México: Editorial McGraw Hill.

Dicaprio, Nicholas S. (1989). "Teorías de la personalidad". México D.F.: Editorial McGrawHill.

Gibson, Jane. Ivancevich, Jhon Y Donnelly, James, (1995). Administración. Editorial IRWIN. Madrid. 
Grados Espinoza, Jaime A. (2,006). Toma de Decisiones: Dinámica de aprendizaje, autoconocimientos y comprensión. Editorial Triallas S.A. 1era Edición.

Grant, R., (2006). Dirección Estratégica. Editorial Civitas. España.

Harrington, H. James. (1993) Cómo Incrementar la Calidad-Productividad en su Empresa. Editorial Public-Mex S.A. México.

Hellriegel, Don; Slocum, John W. Jr.; Woodman, Richard W. (1998). "Comportamiento Organizacional". 8a Edición. México: Editorial International Thomson Editores.

Hellriegel, Don., Sussana E, Jackson. Y John W. Slocum (2002). Administración un Enfoque basado en Competencias. Ed. Thomson. México.

Hernández Rodríguez, Sergio (2002). Administración Pensamiento, Proceso, Estrategia y Vanguardia. Ed. McGraw-Hill. México.

Hill, Ch., (2006). Administración Estratégica. Editorial McGraw-Hill. Colombia.

Hitt, Michael A., Duane Ireland R, Hoskisson Robert E. Administration. Estrategia, Conceptos y Competitividad de Globalización. Editores Internacional Thomson Editores 3era Edición.

Kanawaty, George. (1998). "Introducción al Estudio del Trabajo". Edición Español. México: Editorial McGraw Hill.

Koontz, Harold; Weihrich, Heinz. (1998). "Administración, Una Perspectiva Global". $11^{\text {a }}$ Edición. México: Editorial Mc Graw Hill. México.

Riggs, James L. (2002). "Sistemas de Producción Planeación, Análisis y Control”. $3^{a}$. Edición. México: Editorial Limusa Wiley.

Robbins, Stephen P. (2004). "Comportamiento Organizacional”. 10 Edición. México: Editorial Pearson Prentice Hall.

Schultz, D.P. (1991). "Psicología Industrial". $3^{a}$ Edición. México: Editorial McGraw Hill.

Solano, Ricardo F. (1993). "Administración de Organizaciones". Buenos Aires: Ediciones Interoceánicas S.A. 